\title{
Stable rationality of Brauer-Severi surface bundles
}

Kresch, Andrew ; Tschinkel, Yuri

\begin{abstract}
For sufficiently ample linear systems on rational surfaces we show that a very general associated Brauer-Severi surface bundle is not stably rational.
\end{abstract}

DOI: https://doi.org/10.1007/s00229-018-1087-z

Posted at the Zurich Open Repository and Archive, University of Zurich ZORA URL: https://doi.org/10.5167/uzh-159853

Journal Article

Accepted Version

Originally published at:

Kresch, Andrew; Tschinkel, Yuri (2020). Stable rationality of Brauer-Severi surface bundles. Manuscripta Mathematica, 161(1-2):1-14.

DOI: https://doi.org/10.1007/s00229-018-1087-z 


\title{
STABLE RATIONALITY IN SMOOTH FAMILIES OF THREEFOLDS
}

\author{
BRENDAN HASSETT, ANDREW KRESCH, AND YURI TSCHINKEL
}

ABSTRACT. We exhibit families of smooth projective threefolds with both stably rational and non stably rational fibers.

\section{INTRODUCTION}

Rationality, and thus as well stable rationality, is a deformation invariant property of smooth complex projective curves and surfaces. We now know that rationality and stable rationality specialize in families of smooth complex projective varieties of arbitrary dimension [23], [17]. In dimension at least four, there exist families of smooth complex projective varieties with both rational and non stably rational fibers [14], [15], [27], [28]. The case of relative dimension three is open.

In this note, we exhibit a family of smooth complex threefolds with both stably rational and non stably rational fibers.

Theorem 1. There exists a smooth projective family $\psi: \mathcal{V} \rightarrow B$ of complex threefolds over a connected curve $B$, such that for some $b_{0} \in B$ the fiber $\mathcal{V}_{b_{0}}:=\psi^{-1}\left(b_{0}\right)$ is stably rational and the very general fiber $\mathcal{V}_{b}:=\psi^{-1}(b)$ is not stably rational. In particular, stable rationality is not a deformation invariant of smooth complex projective threefolds.

Our examples originate from the first examples of non-rational but stably rational varieties [7]. The key ingredient is a class of smooth projective surfaces over non-closed fields $k$ that are stably rational but not rational (see Section 2). For $k=\mathbb{C}(t)$, we obtain fibered threefolds $Y \rightarrow \mathbb{P}^{1}$ that are stably rational over $\mathbb{C}$. Using the technique of intermediate Jacobians, one can show that some of these fibered threefolds are not rational.

We work in a similar vein, considering threefolds fibered in stably rational surfaces. Instead of the intermediate Jacobian, we employ the groundbreaking work on specialization of stable rationality by Voisin [32], and its subsequent developments in [9], [30], [13], [16].

Date: March 6, 2018. 
Here is a more detailed summary of the contents of this paper: We review the key class of stably rational non-rational surfaces in Section 2 and recast their Galois-theoretic properties, when defined over $\mathbb{C}(t)$, in terms of finite covers of nodal curves in Section 3. Section 4 sketches the construction of the families of threefolds. The analysis of Section 5 may be of independent interest: How can we construct families of standard conic bundles over a prescribed family of ramification data? Section 6 establishes the failure of stable rationality for general deformations of the examples constructed previously. Finally, we explain why these tools fail to yield stably rational cubic threefolds in Section 7.

Acknowledgments: The first author was partially supported by NSF grants 1551514 and 1701659 and the third author by NSF grant 1601912 . We are grateful to Dan Abramovich and Jean-Louis Colliot-Thélène for discussions about this work.

\section{RECOLLECTIONS ON STABLY RATIONAL NON-RATIONAL SURFACES}

Let $k$ be a field of characteristic zero with absolute Galois group $G_{k}$. For us, a Châtelet surface is

$$
V=\left\{(x, y, z) \mid y^{2}-a z^{2}=f(x)\right\} \subset \mathbb{A}^{3},
$$

where $f \in k[x]$ is a cubic polynomial with Galois group the symmetric group $\mathfrak{S}_{3}$ and $a=\operatorname{disc}(f)$. In particular, $a$ is not a square in $k$. These exist whenever $k$ admits extensions with Galois group $\mathfrak{S}_{3}$.

Let $F(x, w) \in k[x, w]$ be a homogeneous quartic form with $F(x, 1)=$ $f(x)$; note that $w \mid F(x, w)$. The compactification

$$
\hat{V}=\left\{(w: x: y: z) \mid y^{2}-a z^{2}=F(x, w)\right\} \subset \mathbb{P}(1,1,2,2)
$$

has two ordinary singularities $(0: 0: \pm \sqrt{a}: 1)$. This admits a natural embedding as a complete intersection of two quadrics in $\mathbb{P}^{4}$. Writing

$$
u_{0}=x^{2}, \quad u_{1}=x w, \quad u_{2}=w^{2},
$$

we may express

$$
\mathbb{P}(1,1,2,2)=\left\{u_{1}^{2}=u_{0} u_{2}\right\} \subset \mathbb{P}_{u_{0}, u_{1}, u_{2}, y, z}^{4},
$$

so that $\hat{V} \subset \mathbb{P}(1,1,2,2)$ is cut out by a quadratic form. Let $\tilde{V} \rightarrow \hat{V}$ denote the resolution obtained by blowing up the two singularities, which admits a conic fibration with four degenerate fibers

$$
\tilde{V} \rightarrow \mathbb{P}_{x, w}^{1} .
$$


Theorem 2. [7], [29] The Châtelet surface $V$ is stably rational but not rational over $k$.

Remarkably, essentially all stably rational surfaces with these invariants arise in this way:

Theorem 3. [19, Thm. E] Let $W$ be a quartic del Pezzo surface over $k$ such that $\operatorname{Pic}(W) \simeq \mathbb{Z} \oplus \mathbb{Z}$ and $W$ is stably rational but not rational. Then $W$ is birational over $k$ to a Châtelet surface.

The argument involves classifying possible Galois actions, which are constrained by the fact that any stably rational surface $W$ satisfies the following condition: the Néron-Severi group $\mathrm{NS}(\bar{W})$ is a direct summand of a permutation Galois module. This implies that for each closed subgroup $H \subset G_{k}$, the Galois cohomology

$$
H^{1}(H, \mathrm{NS}(\bar{W}))=0 .
$$

Such actions have been classified for degree 4 del Pezzo surfaces in [19, p. 15] and for degrees 3, 2, and 1 in [31]. When the surface admits a conic bundle structure $W \rightarrow \mathbb{P}^{1}$ over $k$ then the Galois action can be described as follows:

Generally, a conic bundle with $n$ degenerate fibers has Galois group contained in the Weyl group $\mathrm{W}\left(D_{n}\right)$, realized as permutations of the irreducible components of the degenerate fibers. We express this as the subgroup of signed permutations having an even number of minus signs, i.e., as a subgroup of

$$
(\mathbb{Z} / 2 \mathbb{Z})^{n} \rtimes \mathfrak{S}_{n}
$$

Here $c_{i}$, for $i=1, \ldots, n$, denotes the identity permutation with minus sign in the $i$ th position, i.e., exchanging the components of the $i$ th fiber.

The case of interest to us is $n=4$ : For Châtelet surfaces as in (2.1), the relevant fibration is $\tilde{V} \rightarrow \mathbb{P}_{x, w}^{1}$. The Galois action on exceptional curves in the fibers corresponds to a representation

$$
\rho: G_{k} \rightarrow \mathrm{W}\left(D_{4}\right)
$$

with image

$$
\left\langle(23) c_{1} c_{2} c_{3} c_{4},(34) c_{1} c_{2} c_{3} c_{4}\right\rangle
$$

which is isomorphic to $\mathfrak{S}_{3}$. This reflects the fact that the discriminant quadratic extension of the cubic $f$ splits the components of each degenerate fiber.

We return to our discussion of Theorem 3: Any standard minimal conic bundle $W \rightarrow \mathbb{P}^{1}$ with four degenerate fibers, satisfying the Galoistheoretic conditions above has Galois representation $\rho$. Such $W$ map 
anticanonically either to quartic del Pezzo surfaces or to Châtelet surfaces in $\mathbb{P}^{4}$. But there always exists a birational map over $k$

$$
W \stackrel{\sim}{\stackrel{\sim}{\rightarrow}} \tilde{V} \text {. }
$$

Our approach exploits the flexibility of passing between these models.

\section{Geometric analysis of Galois Representation}

We now assume $k=\mathbb{C}(t)$. In contrast to the approach of [7], we work with the surfaces $W \rightarrow \mathbb{P}^{1}$ with Galois action $\rho$ as in Section 2, rather than just the Châtelet model. Here we interpret the properties of $\rho$ in geometric terms.

We consider models for $W$, regarding $\mathbb{C}(t)$ as the function field of $\mathbb{P}^{1}$. These are conic bundles over ruled surfaces

$$
\pi: X \rightarrow \mathbb{F} \stackrel{\varphi}{\rightarrow} \mathbb{P}^{1}
$$

satisfying the following:

- $X$ and $\mathbb{F}$ are smooth and projective;

- $\mathbb{F} \rightarrow \mathbb{P}^{1}$ is generically a $\mathbb{P}^{1}$-bundle and $X \rightarrow \mathbb{F}$ is a conic bundle;

- the degeneracy locus of the conic bundle is a nodal curve $D \subset \mathbb{F}$ with smooth irreducible components $C$ and $L$, with $L$ a section and $C$ a simply-branched trisection of $\varphi$;

- the associated double cover $\tilde{D} \rightarrow D$, parametrizing irreducible components of the degenerate fibers of $W \rightarrow \mathbb{P}^{1}$, ramifies over $C \cap L$;

- the Galois action of $\tilde{D} \rightarrow \mathbb{P}^{1}$ coincides with $\rho$.

We recall, by [5, Thm. 1] and [26, Thm. 5.7], over a smooth projective rational surface such as $\mathbb{F}$, the data of a nodal discriminant curve and, for each component, a nontrivial degree 2 cover ramified precisely over the nodes, determine a standard conic bundle, uniquely up to birational isomorphism.

We express the Galois conditions in geometric terms: Let $g$ be the genus of $C, r_{1}, \ldots, r_{2 g+4} \in C$ the ramification points of $C \rightarrow \mathbb{P}^{1}$, $p_{1}, \ldots, p_{2 g+4} \in C$ the residual points in each fiber, $\tilde{C} \rightarrow C$ the discriminant cover, and $p_{1}^{\prime}, \ldots, p_{2 g+4}^{\prime} \in \tilde{C}$ its ramification points. Let $\tilde{L} \rightarrow L$ be the double cover, $w_{1}, \ldots, w_{2 g+4}$ its branch points, and $w_{1}^{\prime}, \ldots, w_{2 g+4}^{\prime} \in \tilde{L}$ the ramification points. The cover $\tilde{D} \rightarrow D$ is the admissible double cover

$$
\tilde{L} \cup_{w_{i}^{\prime}=p_{i}^{\prime}} \tilde{C} \rightarrow L \cup_{w_{i}=p_{i}} C .
$$


In particular,

$$
C \cap L=\left\{p_{1}=w_{1}, \ldots, p_{2 g+4}=w_{2 g+4}\right\}=:\left\{q_{1}, \ldots, q_{2 g+4}\right\} .
$$

Conversely, conic fibrations $X \rightarrow \mathbb{F}$ with degeneracy (i.e., ramification) data as above necessarily have Galois representation $\rho$, as the action on the Néron-Severi group can be read off from the induced permutation of the irreducible components of the four degenerate conic fibers.

Given such a $\pi: X \rightarrow \mathbb{F} \rightarrow \mathbb{P}^{1}$, the fiber $W$ over the generic point of $\mathbb{P}^{1}$ is as in Section 2 , thus is stably rational over $\mathbb{C}(t)$ by Theorem 3 . It follows that $X$ is stably rational over $\mathbb{C}$.

Remark 4. The key constraint is that the images of the branch loci of $\tilde{C} \rightarrow C$ and $\tilde{L} \rightarrow L$ in $\mathbb{P}^{1}$ coincide

$$
\varphi\left(\left\{p_{1}, \ldots, p_{2 g+4}\right\}\right)=\varphi\left(\left\{w_{1}, \ldots, w_{2 g+4}\right\}\right) .
$$

Once $C \rightarrow \mathbb{P}^{1}$ is given, there is a canonical choice of $\tilde{L} \rightarrow L$, determined by the discriminant quadratic extension.

Remark 5. The double cover $\tilde{D} \rightarrow D$ is admissible and its Prym variety $P$ is a principally polarized abelian variety of dimension $3 g+$ 2. If $g>0$ then $P$ is not a product of Jacobians [7, Rem. 7]. The intermediate Jacobian $\mathrm{IJ}(X)=P$. Hence $X$ is not rational.

\section{Construction of examples}

4.1. Embedding of the degeneracy curve. We use the machinery of Section 3 to construct examples of stably rational threefolds. The simplest possible case of interest is $g=1$.

We start by fixing $f: C \rightarrow \mathbb{P}^{1}$, a simply branched triple cover, with ramification points $r_{1}, \ldots, r_{6}$ and residual points $p_{1}, \ldots, p_{6}$. In other words, $p_{i}$ is the residual to $r_{i}$ in $f^{-1}\left(f\left(r_{i}\right)\right)$. Set $L=\mathbb{P}^{1}$ and glue $p_{i}$ to $f\left(r_{i}\right)$ to obtain $D$. We use $q_{i}, i=1, \ldots, 6$, for the nodes of $D$ arising from gluing $p_{i}$ and $f\left(r_{i}\right)$. Let $\varphi: D \rightarrow \mathbb{P}^{1}$ denote the resulting degree 4 cover.

Our goal is to embed $D$ in the simplest possible rational surface. We may interpret $D$ as a stable curve of genus six.

Remark 6. We show in Section 7 that $D$ cannot be embedded as a quintic plane curve. Thus our approach does not yield stably rational cubic threefolds. Recall that projecting from a line in a cubic threefold gives a conic bundle over $\mathbb{P}^{2}$ with quintic degeneracy locus, and almost all quintic plane curves arise in this way. 
The generic stable curve $D^{\prime}$ of genus six arises as a bi-anticanonical curve in a quintic del Pezzo surface $S$. Realizing $S$ as the blowup of $\mathbb{P}^{2}$ in four points, $D^{\prime}$ may be interpreted as a sextic plane curve with four nodes. This motivates the following technical result:

Proposition 7. Let $D$ be as above. Then there exist:

- an embedding $C \hookrightarrow \mathbb{P}^{2}$ as a cubic plane curve;

- a morphism $\iota: L \rightarrow \mathbb{P}^{2}$ birational onto a cubic curve singular at $s_{4}$;

satisfying the following:

- projection from $s_{4}$

$$
\mathbb{P}^{2} \rightarrow \mathbb{P}^{1}
$$

restricts to $\varphi$ on $C$;

- the intersection

$$
C \cap \iota(L) \supset\left\{q_{1}, \ldots, q_{6}\right\}
$$

and the residual points of intersection $s_{1}, s_{2}, s_{3}$ are collinear.

Proof. The embedding of $C$ arises from the linear series associated with $\varphi^{*} \mathcal{O}_{\mathbb{P}^{1}}(1) \mid C$. Let $s_{4} \in \mathbb{P}^{2}$ be the point inducing the triple cover $C \rightarrow \mathbb{P}^{1}$.

We recall some classical terminology [10]: The ramification of $C \rightarrow$ $\mathbb{P}^{1}$ is given by the intersection of $C$ with its polar conic with respect to $s_{4}$. The six residual points are given by the intersection of $C$ with its satellite conic with respect to $s_{4}[10$, p. 157].

Choose a cubic plane curve $C_{\text {sing }}$ with double point at $s_{4}$ and containing the intersection of $C$ with its satellite conic. We claim this is irreducible. Otherwise, one of its irreducible components would have to be the satellite conic, which is precluded by:

Lemma 8. Fix a smooth plane cubic $C \subset \mathbb{P}^{2}$ and a point $p \in \mathbb{P}^{2} \backslash C$. Then the satellite conic for $C$ with respect to $p$ does not contain $p$.

Proof. Let $P_{p}(C)$ be the polar conic, $S_{p}(C)$ the satellite conic, and $P_{p}\left(P_{p}(C)\right)$ the polar line of the polar conic, which joins the two ramification points of the double cover

$$
P_{p}(C) \rightarrow \mathbb{P}^{1}
$$

induced by projection from $p$.

By [10, p. 157, Exer. 3.19], we have

$$
Z:=S_{p}(C) \cap P_{p}(C)
$$


consists of two points, each of multiplicity two, which coincide with

$$
P_{p}\left(P_{p}(C)\right) \cap P_{p}(C) .
$$

There is a unique plane conic containing $Z$ and $p$ - the union of the two tangents to $S_{p}(C)$ through $p$. The same conclusion can be obtained through a direct computation of the equations for $S_{p}(C)$ and $P_{p}(C)$ in [22, Example 2.2]. This proves the Lemma.

We return to the argument for Proposition 7. Fix $\iota: L \rightarrow C_{\text {sing }} \subset \mathbb{P}^{2}$ to be normalization. The base locus of the pencil of cubics $\left\langle C, C_{\text {sing }}\right\rangle$ has six points failing to impose independent conditions on quadrics. The residual three points $\left\{s_{1}, s_{2}, s_{3}\right\}$ fail to impose independent conditions on linear forms, hence are collinear.

For our application, we want $C_{\text {sing }}$ to be a nodal cubic. A concrete example over a finite field shows that this is possible.

Example 9. We work over the finite field $\mathbf{F}_{p}$, with $p=13$. Let $C$ be the cubic curve

$x^{3}+5 x^{2} y+12 x^{2} z+7 x y^{2}+7 x y z+3 x z^{2}+10 y^{3}+y^{2} z+2 y z^{2}+6 z^{3}=0$.

Put

$$
s_{4}:=(1: 0: 0) \in \mathbb{P}^{2}\left(\mathbf{F}_{p}\right)
$$

and consider the satellite conic associated to $C$ and $s_{4}$. The intersection points with $C$ (residual points for the projection from $s_{4}$ ) are:

$$
\{(4: 10: 1),(7: 12: 1),(6: 1: 1),(2: 7: 1),(2: 4: 1),(4: 1: 0)\} .
$$

The cubic curve $L$, given by

$$
x y^{2}+2 x y z+11 x z^{2}+9 y^{3}+y^{2} z+10 y z^{2}=0,
$$

is nodal at $s_{4}$ and passes through the residual points in (4.1).

Let $S_{0}$ denote the blowup of $\mathbb{P}^{2}$ at four points $\left\{s_{1}, s_{2}, s_{3}, s_{4}\right\}$, where $s_{1}, s_{2}, s_{3}$ are collinear and $s_{4}$ is generic. Projection from $s_{4}$ induces a morphism

$$
\Phi: S_{0} \rightarrow \mathbb{P}^{1} .
$$

Proposition 7 gives an embedding $D \hookrightarrow S_{0}$ as a bi-anticanonical curve. 
4.2. The family of surfaces. Let $\mathcal{S} \rightarrow B$ be a smooth projective family of surfaces, where $S_{0}=\mathcal{S}_{b_{0}}$ is the degenerate quintic del Pezzo surface as in Section 4.1 and $\mathcal{S}_{b}, b \neq b_{0}$ is a smooth quintic del Pezzo surface $S$. The deformation space of $S_{0}$ is smooth, and the dimension of the bi-anticanonical linear series remains constant in the family. So, there exists a family of nodal curves $\mathcal{D} \rightarrow B$ embedded in $\mathcal{S}$ over $B$, where $\mathcal{D}_{0}$ is as constructed in Section 4.1, and $\mathcal{D}_{b}$ is smooth for $b \neq b_{0}$.

4.3. The conic bundles in the family. The analysis of Section 3 implies that a conic bundle $X_{0} \rightarrow S_{0}$ with ramification data given by $\tilde{D} \rightarrow D$ is stably rational. Indeed, $\Phi \mid D=\varphi$, by construction, thus the generic fiber of the composition

$$
X_{0} \rightarrow S_{0} \rightarrow \mathbb{P}^{1}
$$

is a stably rational degree 4 del Pezzo surface over $\mathbb{C}\left(\mathbb{P}^{1}\right)$. Such an $X_{0}$ is necessarily stably rational over $\mathbb{C}$.

Let $X \rightarrow S$ denote a standard conic bundle degenerate over a generic bi-anticanonical divisor in $S$. The challenge is to fit the conic bundles $X$ and $X_{0}$ into a family

$$
\varpi: \mathcal{X} \rightarrow \mathcal{S} \rightarrow B
$$

Indeed, we make choices in constructing a standard conic bundle from its ramification data - the resulting threefold is unique only up to birational equivalence, although the birational maps between the various models are well-understood $[25,26]$. It remains to show that these choices can be made coherently in one-parameter families; Theorem 17 shows this is possible.

\section{Deformation of CONiC BUndLeS}

Let $k$ be an algebraically closed field of characteristic different from 2 and $\left(B, b_{0}\right)$, a pointed curve. We consider a smooth projective family of rational surfaces $\mathcal{S} \rightarrow B$, irreducible divisor $\mathcal{D} \subset \mathcal{S}$, smooth except for ordinary double points at $q_{1}, \ldots, q_{r}$ in the fiber $\mathcal{S}_{b_{0}}$, and a degree 2 covering $\widetilde{\mathcal{D}} \rightarrow \mathcal{D}$, unramified away $q_{1}, \ldots, q_{r}$ with $\widetilde{\mathcal{D}}$ smooth. We assume that $\mathcal{D}_{b_{0}}$ has a node at $q_{1}, \ldots, q_{r}$ and is otherwise smooth.

The goal is to show (Theorem 17 in Section 5.4) that after replacing $\left(B, b_{0}\right)$ by an étale neighborhood there is a smooth family of standard conic bundles $\mathcal{V} \rightarrow B$ such that the birational type of the fiber over every point $b \in B$ corresponds to the ramification data $\widetilde{\mathcal{D}}_{b} \rightarrow \mathcal{D}_{b}$. 
5.1. An algebraic group and some of its representations. Working over $\operatorname{Spec}(\mathbb{Z})$, we consider the classifying stack $B \mathbb{Z} / 2 \mathbb{Z}$ with étale cover

$$
\pi: \operatorname{Spec}(\mathbb{Z}) \rightarrow B \mathbb{Z} / 2 \mathbb{Z}
$$

sending a scheme $T$ to the trivial torsor $T \times \mathbb{Z} / 2 \mathbb{Z} \rightarrow T$.

The stack $B \mathbb{G}_{m}$ classifies $\mathbb{G}_{m}$-torsors, or equivalently, line bundles.

A general construction is the restriction of scalars along a proper flat morphism of finite presentation, applicable to algebraic stacks, locally of finite presentation with affine diagonal [11]. We need $\pi_{*} B \mathbb{G}_{m}$, the restriction of scalars of $B \mathbb{G}_{m}$ under the morphism $\pi$ :

$$
\pi_{*} B \mathbb{G}_{m} \cong B H, \quad H:=\left\{\left(\begin{array}{ll}
* & 0 \\
0 & *
\end{array}\right)\right\} \cup\left\{\left(\begin{array}{ll}
0 & * \\
* & 0
\end{array}\right)\right\} \subset G L_{2} .
$$

Indeed, an $H$-torsor $E \rightarrow T$ determines a $\mathbb{Z} / 2 \mathbb{Z}$-torsor $S \rightarrow T$ (connected components of fibers), such that $T \times{ }_{S} E$ admits a canonical reduction of structure group to $\mathbb{G}_{m} \times \mathbb{G}_{m}$, i.e., is determined by a pair of line bundles $\left(L, L^{\prime}\right)$ on $S$; to $E \rightarrow T$ we associate $S \rightarrow T$ and $L$. Conversely, given $S \rightarrow T$ and $L$ and letting $\sigma: S \rightarrow S$ denote the covering involution, we associate to $\left(L, \sigma^{*} L\right)$ a $\mathbb{G}_{m} \times \mathbb{G}_{m}$-torsor over $S$, which we recognize as having the structure of $H$-torsor over $T$.

Let $N$ denote the homomorphism $H \rightarrow \mathbb{G}_{m}$ given by multiplication of the nonzero matrix entries. We define $G$ to be the kernel of $N$ :

$$
1 \rightarrow G \rightarrow H \stackrel{N}{\rightarrow} \mathbb{G}_{m} \rightarrow 1 .
$$

Correspondingly we have

$$
\pi_{*} B \mathbb{G}_{m} \cong B H \rightarrow B \mathbb{G}_{m}
$$

sending $\mathbb{Z} / 2 \mathbb{Z}$-torsor $S \rightarrow T$ with line bundle $L$ on $S$ to the norm $N_{T / S}(L)$. So, $B G$ classifies $\mathbb{Z} / 2 \mathbb{Z}$-torsors with line bundle and trivialization of the norm line bundle. To give a $G$-torsor over a scheme $T$ is the same as to give a $\mathbb{Z} / 2 \mathbb{Z}$-torsor $S \rightarrow T$, a line bundle $L$ on $S$, and an isomorphism $L \otimes \sigma^{*} L \cong \mathcal{O}_{S}$, invariant under the covering involution $\sigma: S \rightarrow S$.

The 2-dimensional representation of $H$, given by $H \subset G L_{2}$, associates to the $H$-torsor determined by $S \rightarrow T$ (with covering involution $\sigma$ ) and $L$, the rank 2 vector bundle over $T$, obtained by descent un$\operatorname{der} S \rightarrow T$ from $L \oplus \sigma^{*} L$ over $S$ with $\sigma^{*}\left(L \oplus \sigma^{*} L\right) \cong L \oplus \sigma^{*} L$. The corresponding 2-dimensional representation of $G$ admits the same description and will be denoted by $\rho_{2}$. 
There is the exact sequence

$$
1 \rightarrow \mu_{2} \rightarrow G \rightarrow G \rightarrow 1
$$

where the homomorphism $G \rightarrow G$ is given by squaring the matrix entries. The representation $\rho_{2}$ of the middle group $G$ in (5.1) determines a projective representation

$$
\omega: G \rightarrow P G L_{2},
$$

of the group $G$ appearing on the right in (5.1); concretely,

$$
\left(\begin{array}{cc}
\alpha & 0 \\
0 & \alpha^{-1}
\end{array}\right) \stackrel{\omega}{\mapsto}\left[\left(\begin{array}{cc}
\alpha & 0 \\
0 & 1
\end{array}\right)\right], \quad\left(\begin{array}{cc}
0 & \beta \\
\beta^{-1} & 0
\end{array}\right) \stackrel{\omega}{\mapsto}\left[\left(\begin{array}{cc}
0 & \beta \\
1 & 0
\end{array}\right)\right] .
$$

As well, $\operatorname{det}\left(\rho_{2}\right)$ and $\rho_{2}^{\vee} \otimes \rho_{2}$ determine linear representations $\chi$, respectively $\rho_{4}$, of the group $G$ on the right. There is the trace homomorphism $\rho_{4} \rightarrow 1$ to a trivial one-dimensional representation.

Proposition 10. Over $\operatorname{Spec}(\mathbb{Z}[1 / 2])$ we have an isomorphism of representations

$$
\rho_{4} \cong 1 \oplus \chi \oplus \rho_{2}
$$

of $G$ such that projection to the first factor gives the trace homomorphism.

Proof. Direct computation.

The kernel $\chi \oplus \rho_{2}$ of the trace homomorphism in Proposition 10 will be denoted by $\rho_{3}$.

Example 11. Let $T$ be a scheme over $\operatorname{Spec}(\mathbb{Z}[1 / 2])$ with $\mathbb{Z} / 2 \mathbb{Z}$-torsor $S \rightarrow T$ and covering involution $\sigma: S \rightarrow S$, and let $S^{\prime} \rightarrow T$ be a second $\mathbb{Z} / 2 \mathbb{Z}$-torsor, to which there is an associated line bundle $L_{0}$ with $L_{0}^{\otimes 2} \cong \mathcal{O}_{T}$. Let $L$ denote the pullback of $L_{0}$ to $S$, so we have $\sigma^{*} L \cong L$, canonically. The identification

$$
L \otimes \sigma^{*} L \cong L^{\otimes 2} \cong \mathcal{O}_{S}
$$

determines a $G$-torsor, which arises from the pair of $\mathbb{Z} / 2 \mathbb{Z}$-torsors under the identification of the subgroup of $G$, generated the $2 \times 2$-permutation matrices and $\mu_{2}$, with the Klein four-group.

5.2. Root stacks and conic bundles. We recall and introduce notation for three flavors of root stack:

(i) root stack $\sqrt{L}$ of a line bundle $L$ on a smooth algebraic variety $S$ over $k$, a smooth Deligne-Mumford stack that is Zariski locally over $S$ isomorphic to a product with the classifying stack $B \mu_{2}$ [8, Defn. 2.2.6] [2, §B.1]; 
(ii) root stack $\sqrt{(S, D)}$ of a divisor $D \subset S$, a Deligne-Mumford stack, locally for $D$ defined by $f=0$ on affine open $\operatorname{Spec}(A)$, given by

$$
\left[\operatorname{Spec}\left(A[z] /\left(z^{2}-f\right)\right) / \mu_{2}\right],
$$

where $\mu_{2}$ acts by scalar multiplication on $z$ [8, Defn. 2.2.1] [2, $\S \mathrm{B} .2]$;

(iii) iterated root stack

$$
\sqrt{\left(S,\left\{D, D^{\prime}\right\}\right)}=\sqrt{(S, D)} \times_{S} \sqrt{\left(S, D^{\prime}\right)}
$$

of a pair of divisors $D, D^{\prime} \subset S$ [8, Defn. 2.2.4] (or more generally of an arbitrary collection $\left\{D_{1}, \ldots, D_{N}\right\}$ of divisors on $S$ ).

The root stack in (ii) is smooth if $D$ is smooth, and in (iii) is smooth if $D$ and $D^{\prime}$ are smooth and intersect transversally (or more generally if $D_{1} \cup \cdots \cup D_{N}$ is a simple normal crossing divisor). It is useful to be aware of a fourth flavor, defined by Matsuki and Olsson [21, Thm. 4.1], which for a normal crossing divisor $D \subset S$ is a smooth DeligneMumford stack with morphism to $S$ that étale locally over $S$ is of the form described in (iii).

Let $S$ be a smooth algebraic variety over $k$ and $D \subset S, D=D_{1} \cup$ $\cdots \cup D_{N}$ a simple normal crossing divisor with $D_{i} \cap D_{i^{\prime}} \cap D_{i^{\prime \prime}}=\emptyset$ for distinct $i, i^{\prime}, i^{\prime \prime}$. One way that a standard conic bundle over $S$ may arise is by modifying a smooth $\mathbb{P}^{1}$-fibration

$$
P \rightarrow \sqrt{\left(S,\left\{D_{1}, \ldots, D_{N}\right\}\right)} .
$$

We assume that the corresponding class in the Brauer group $\operatorname{Br}(S \backslash D)$ is nontrivial, obstructed for every $i$ from extending to a neighborhood of the generic point of $D_{i}$ by the class of a nontrivial degree 2 covering $\widetilde{D}_{i} \rightarrow D_{i}$ that is furthermore assumed to be ramified over the generic point of every component of $D_{i} \cap D_{i^{\prime}}$ for all $i^{\prime} \neq i$. Following [24] and appealing to [18, Rmk. 2.3], associated with (5.2) there is a standard conic bundle $V \rightarrow S$. The construction over $S \backslash D^{\text {sing }}$ is that of [18, Prop. 3.1]: blow up the locus in $P$ with $\mu_{2}$-stabilizer, contract, and descend to $S \backslash D^{\text {sing }}$. There is a unique extension to a standard conic bundle over $S$.

Example 12. When $S=\mathbb{A}^{2}$ and $D=D_{1} \cup D_{2}$, union of coordinate axes, we have

$$
\sqrt{\left(S,\left\{D_{1}, D_{2}\right\}\right)} \cong\left[\mathbb{A}^{2} / \mu_{2} \times \mu_{2}\right]
$$

where, writing $\kappa, \lambda$ for coordinates on $\mathbb{A}^{2}$, the action of the first factor $\mu_{2}$ is by scalar multiplication on $\kappa$ and of the second factor $\mu_{2}$, by scalar 
multiplication on $\lambda$; here, $S$ is identified with $\operatorname{Spec}\left(k\left[\kappa^{2}, \lambda^{2}\right]\right)$. Let

$$
P:=\left[\mathbb{A}^{2} \times \mathbb{P}^{1} / \mu_{2} \times \mu_{2}\right],
$$

where the action of the first factor $\mu_{2}$ on $\mathbb{P}^{1}$ is by permutation of the coordinates and of the second factor $\mu_{2}$, by scalar multiplication on one of the coordinates. The construction described above leads to $V$, defined inside $S \times \mathbb{P}^{2}$ with projective coordinates $x, y, z$ by

$$
\kappa^{2} x^{2}+\lambda^{2} y^{2}-z^{2}=0 .
$$

The smooth $\mathbb{P}^{1}$-fibration and conic bundle are identified over $S \backslash D$ by

$$
(\kappa, \lambda,(\alpha: \beta)) \mapsto\left(\kappa^{2}, \lambda^{2},\left(\lambda\left(-\alpha^{2}+\beta^{2}\right): 2 \kappa \alpha \beta: \kappa \lambda\left(\alpha^{2}+\beta^{2}\right)\right)\right) .
$$

5.3. A useful conic bundle. We work out in detail one instance of the construction of Section 5.2. Let $\mathbb{Z} / 2 \mathbb{Z}$ act on $\mathbb{P}^{1} \times \mathbb{P}^{1}$ by swapping the factors and consider

$$
W:=\left[\mathbb{P}^{1} \times \mathbb{P}^{1} /(\mathbb{Z} / 2 \mathbb{Z})\right] .
$$

Since $\mathbb{P}^{1} \times \mathbb{P}^{1}$ is, by

$$
\left(\left(u^{\prime}: v^{\prime}\right),\left(u^{\prime \prime}: v^{\prime \prime}\right)\right) \mapsto\left(\frac{1}{2}\left(u^{\prime} v^{\prime \prime}+v^{\prime} u^{\prime \prime}\right): u^{\prime} u^{\prime \prime}: v^{\prime} v^{\prime \prime}\right)
$$

a double cover of $\mathbb{P}^{2}$ branched over the conic in $\mathbb{P}^{2}$ defined by $t^{2}=u v$, we may view $W$ as the root stack of $\mathbb{P}^{2}$ along the conic. Let $H$ be the line $v=0$ in $\mathbb{P}^{2}$ and

$$
X:=W \times_{\mathbb{P}^{2}} \sqrt{\mathcal{O}_{\mathbb{P}^{2}}(-H)} .
$$

The root stack $\sqrt{\mathcal{O}_{\mathbb{P}^{2}}(-H)}$ carries a tautological line bundle whose tensor square is identified with the pulllback of $\mathcal{O}_{\mathbb{P}^{2}}(-H)$. The tautological line bundle, pulled back to $X$, will be denoted by $M$; its pullback to the degree 2 étale cover $\left(\mathbb{P}^{1} \times \mathbb{P}^{1}\right) \times_{\mathbb{P}^{2}} \sqrt{\mathcal{O}_{\mathbb{P}^{2}}(-H)}$ will be denoted by $M^{\prime}$. The pre-image of $H$ in $\mathbb{P}^{1} \times \mathbb{P}^{1}$ is a union $H^{\prime} \cup H^{\prime \prime}$, where $H^{\prime}$ is defined by $v^{\prime}=0$ and $H^{\prime \prime}$, by $v^{\prime \prime}=0$. Now we let

$$
L:=M^{\prime} \otimes_{\mathcal{O}_{\mathbb{P}^{1} \times \mathbb{P}^{1}}} \mathcal{O}_{\mathbb{P}^{1} \times \mathbb{P}^{1}}\left(H^{\prime}\right) .
$$

So $\sigma^{*} L \cong M^{\prime} \otimes_{\mathcal{O}_{\mathbb{P}} \times \mathbb{P}^{1}} \mathcal{O}_{\mathbb{P}^{1} \times \mathbb{P}^{1}}\left(H^{\prime \prime}\right)$, where $\sigma$ denotes the covering involution, and

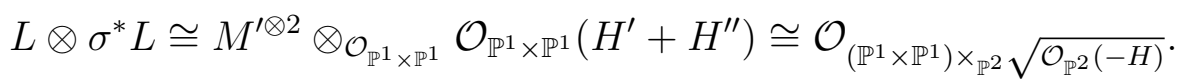

These data determine a $G$-torsor over $X$, hence via the representation $\omega$ a smooth $\mathbb{P}^{1}$-fibration

$$
F \rightarrow X
$$


By base change to the line bundle $M$ we obtain

$$
P:=F \times_{X} M \rightarrow M
$$

Proposition 13. We write $t_{0}, u_{0}, v_{0}$ for affine coordinates on $\mathbb{A}^{3}$ and view the blow-up $B \ell_{0} \mathbb{A}^{3}$ as subvariety of $\mathbb{A}^{3} \times \mathbb{P}^{2}$, where $\mathbb{P}^{2}$ has projective coordinates $t, u, v$.

(i) Let $D \subset \mathbb{A}^{3}$ be the divisor $u_{0} v_{0}-t_{0}^{2}=0$. Then

$$
M \cong \sqrt{\left(B \ell_{0} \mathbb{A}^{3},\left\{D^{\prime}, E\right\}\right)}
$$

where $D^{\prime}$ denotes the proper transform of $D$ and $E$, the exceptional divisor.

(ii) Let us write $\theta$ for the canonical section of $\mathcal{O}_{B \ell_{0} \mathbb{A}^{3}}(-1)$, vanishing on E. The conic bundle construction of Section 5.2 applied to (5.3) yields the conic bundle

$$
V \subset \mathbb{P}\left(\mathcal{O}_{B \ell_{0} \mathbb{A}^{3}}(1) \oplus \mathcal{O}_{B \ell_{0} \mathbb{A}^{3}} \oplus \mathcal{O}_{B \ell_{0} \mathbb{A}^{3}}\right)
$$

defined by the symmetric morphism of vector bundles

$$
\begin{array}{r}
\left(\mathcal{O}_{B \ell_{0} \mathbb{A}^{3}}(1) \oplus \mathcal{O}_{B \ell_{0} \mathbb{A}^{3}} \oplus \mathcal{O}_{B \ell_{0} \mathbb{A}^{3}}\right) \otimes \mathcal{O}_{B \ell_{0} \mathbb{A}^{3}}(-1) \stackrel{\left(\begin{array}{ccc}
\theta & 0 & 0 \\
0 & -u & -t \\
0 & -t & -v
\end{array}\right)}{\longrightarrow} \\
\mathcal{O}_{B \ell_{0} \mathbb{A}^{3}}(-1) \oplus \mathcal{O}_{B \ell_{0} \mathbb{A}^{3}} \oplus \mathcal{O}_{B \ell_{0} \mathbb{A}^{3}} .
\end{array}
$$

Proof. Assertion (i) follows directly from the identification of $B \ell_{0} \mathbb{A}^{3}$ with the total space of $\mathcal{O}_{\mathbb{P}^{2}}(-H)$ and two general facts about root stacks: the root stack constructions (i)-(iii) of Section 5.2 commute with base change, and the total space of the tautological line bundle on the root stack of a line bundle is isomorphic to the root stack of the zero section as divisor in the original line bundle.

It suffices to identify the outcome of the conic bundle construction of Section 5.2 with the conic bundle defined by the matrix in (ii) over the complement of a curve in $B \ell_{0} \mathbb{A}^{3}$. We do this over the union of two affine charts, one defined by the nonvanishing of $v$ and the other, by the nonvanishing of $u$.

Chart 1: coordinates $v_{0}, t_{1}, u_{1}$ with $t_{0}=v_{0} t_{1}$ and $u_{0}=v_{0} u_{1}$. The corresponding open substack of $M$ is isomorphic to

$$
\left[\mathbb{A}^{2} /(\mathbb{Z} / 2 \mathbb{Z})\right] \times\left[\mathbb{A}^{1} /(\mathbb{Z} / 2 \mathbb{Z})\right]
$$


action by swapping the coordinates and multiplication by -1 of the coordinate on respective factors. Denoting coordinates by $u^{\prime}, u^{\prime \prime}$, respectively $\lambda_{1}$, we have

$$
v_{0}=\lambda_{1}^{2}, \quad t_{1}=\frac{1}{2}\left(u^{\prime}+u^{\prime \prime}\right), \quad u_{1}=u^{\prime} u^{\prime \prime} .
$$

We are in the situation of Example 11 , where the first $\mathbb{Z} / 2 \mathbb{Z}$-torsor is $\mathbb{A}^{2} \times\left[\mathbb{A}^{1} /(\mathbb{Z} / 2 \mathbb{Z})\right]$ and the second $\mathbb{Z} / 2 \mathbb{Z}$-torsor is $\left[\mathbb{A}^{2} /(\mathbb{Z} / 2 \mathbb{Z})\right] \times \mathbb{A}^{1}$. So the corresponding open substack of $P$ is isomorphic to

$$
\left[\mathbb{A}^{2} \times \mathbb{A}^{1} \times \mathbb{P}^{1} /(\mathbb{Z} / 2 \mathbb{Z} \times \mathbb{Z} / 2 \mathbb{Z})\right]
$$

with action of first $\mathbb{Z} / 2 \mathbb{Z}$ factor by swapping the coordinates of $\mathbb{A}^{2}$ and swapping the coordinates of $\mathbb{P}^{1}$, and action of second $\mathbb{Z} / 2 \mathbb{Z}$ factor by multiplication by -1 of the coordinate of $\mathbb{A}^{1}$ and multiplication by -1 of one of the coordinates of $\mathbb{P}^{1}$.

Chart 2: coordinates $u_{0}, t_{2}, v_{2}$ with $t_{0}=u_{0} t_{2}$ and $v_{0}=u_{0} v_{2}$. The corresponding open substacks of $M$ and of $P$ admit the same description as in Chart 1 . With coordinates $v^{\prime}, v^{\prime \prime}$ and $\lambda_{2}$ on respective factors of $M \cong\left[\mathbb{A}^{2} /(\mathbb{Z} / 2 \mathbb{Z})\right] \times\left[\mathbb{A}^{1} /(\mathbb{Z} / 2 \mathbb{Z})\right]$, we have

$$
u_{0}=\lambda_{2}^{2}, \quad t_{2}=\frac{1}{2}\left(v^{\prime}+v^{\prime \prime}\right), \quad v_{2}=v^{\prime} v^{\prime \prime} .
$$

Transition between charts: Given a $k$-scheme $T$, the data of a $T$ valued point of Chart 1 consist of a $\mathbb{Z} / 2 \mathbb{Z}$-torsor $S \rightarrow T$ with equivariant map $S \rightarrow \mathbb{A}^{2}$ and a second $\mathbb{Z} / 2 \mathbb{Z}$-torsor $S^{\prime} \rightarrow T$ with equivariant map $S^{\prime} \rightarrow \mathbb{A}^{1}$. To land in the overlap with Chart 2 the morphism $S \rightarrow \mathbb{A}^{2}$ should have image contained in $\left(\mathbb{A}^{1} \backslash\{0\}\right)^{2}$. The product of coordinates is invariant and thus determines an invertible function $f$ on $T$. We denote by $\sqrt{f} \rightarrow T$ the associated degree 2 étale cover and combine this with $S^{\prime}$ to obtain $\mathbb{Z} / 2 \mathbb{Z}$-torsor

$$
\widetilde{S}^{\prime}:=S^{\prime} \times \mathbb{Z} / 2 \mathbb{Z} \sqrt{f} .
$$

Then, with notation mul and inv for multiplication and multiplicative inverse, respectively, the Chart 2 data consist of $\mathbb{Z} / 2 \mathbb{Z}$-torsors and equivariant maps

$$
S \rightarrow T \quad \text { with } \quad S \rightarrow\left(\mathbb{A}^{1} \backslash\{0\}\right)^{2} \stackrel{\text { inv } \times \text { inv }}{\longrightarrow}\left(\mathbb{A}^{1} \backslash\{0\}\right)^{2} \subset \mathbb{A}^{2}
$$

and

$\widetilde{S}^{\prime} \rightarrow T \quad$ with $\quad \widetilde{S}^{\prime} \rightarrow \mathbb{A}^{1} \quad$ induced by $\quad S^{\prime} \times \sqrt{f} \rightarrow \mathbb{A}^{1} \times \mathbb{G}_{m} \stackrel{\text { mul }}{\rightarrow} \mathbb{A}^{1}$.

Conic bundle: We introduce new coordinate $\kappa_{1}=(1 / 2)\left(-u^{\prime}+u^{\prime \prime}\right)$ on Chart 1 and $\kappa_{2}=(1 / 2)\left(-v^{\prime}+v^{\prime \prime}\right)$ on Chart 2. On Chart 1 we use 
$t_{1}, \kappa_{1}$ in place of $u^{\prime}, u^{\prime \prime}$, and on Chart 2 we use $t_{2}, \kappa_{2}$ in place of $v^{\prime}, v^{\prime \prime}$. We have

$$
u_{1}=t_{1}^{2}-\kappa_{1}^{2} \quad \text { and } \quad v_{2}=t_{2}^{2}-\kappa_{2}^{2} .
$$

Performing the change of coordinates leads to description of open substack of $M$ as

$$
\mathbb{A}^{1} \times\left[\mathbb{A}^{2} /(\mathbb{Z} / 2 \mathbb{Z} \times \mathbb{Z} / 2 \mathbb{Z})\right],
$$

with coordinates $t_{i}$, respectively $\kappa_{i}, \lambda_{i}$ on Chart $i$ for $i \in\{1,2\}$. For the open substack of $P$ over each chart, there is simply an extra factor $\mathbb{P}^{1}$ as in (5.4). We have, over each chart, exactly the situation of Example 12 (up to an extra $\mathbb{A}^{1}$-factor), and the conic bundle is thereby defined by an explicit equation in projective coordinates $x_{i}, y_{i}, z_{i}$. With the change of coordinates

$$
\tilde{z}_{i}=z_{i}-t_{i} x_{i}
$$

we obtain the equation

$-u_{1} x_{1}^{2}+v_{0} y_{1}^{2}-\tilde{z}_{1}^{2}-2 t_{1} x_{1} \tilde{z}_{1}=0, \quad$ resp. $\quad-v_{2} x_{2}^{2}+u_{0} y_{2}^{2}-\tilde{z}_{2}^{2}-2 t_{2} x_{2} \tilde{z}_{2}=0$,

with map

$\left(t_{i}, \kappa_{i}, \lambda_{i},\left(\alpha_{i}: \beta_{i}\right)\right) \mapsto\left(\lambda_{i}\left(-\alpha_{i}^{2}+\beta_{i}^{2}\right): 2 \kappa_{i} \alpha_{i} \beta_{i}:\left(\kappa_{i}+t_{i}\right) \lambda_{i} \alpha_{i}^{2}+\left(\kappa_{i}-t_{i}\right) \lambda_{i} \beta_{i}^{2}\right)$.

Writing $\gamma$ for a square root of $u_{1}=u^{\prime} u^{\prime \prime}$, we have

$$
\lambda_{2}=\gamma \lambda_{1} \quad \text { and } \quad\left(\alpha_{2}: \beta_{2}\right)=\left(u^{\prime-1} \gamma \alpha_{1}: \beta_{1}\right),
$$

and it follows that the projective coordinates on the two charts are related by

$$
\left(x_{2}: y_{2}: \tilde{z}_{2}\right)=\left(\tilde{z}_{1}: u_{1}^{-1} y_{1}: x_{1}\right) .
$$

The conic bundle defined by the matrix in (ii) yields, on the two charts, precisely the equations obtained above with relation (5.5) between projective coordinates.

Given a smooth variety, the elementary transformation of a projectivized vector bundle along a section over a divisor is the outcome of blowing up the section and contracting to the projectivization of a vector bundle, whose dual is in a natural way a subsheaf of the dual of the original vector bundle [20].

Proposition 14. We adopt the notation of Proposition 13. The elementary transformation of

$$
\mathbb{P}\left(\mathcal{O}_{B \ell_{0} \mathbb{A}^{3}}(1) \oplus \mathcal{O}_{B \ell_{0} \mathbb{A}^{3}} \oplus \mathcal{O}_{B \ell_{0} \mathbb{A}^{3}}\right)
$$


along the section $\mathbb{P}\left(\mathcal{O}_{E}(1) \oplus 0 \oplus 0\right)$ over $E$ is $B \ell_{0} \mathbb{A}^{3} \times \mathbb{P}^{2}$. Writing $x, y, z$ for projective coordinates on the $\mathbb{P}^{2}$ factor, the conic bundle $V \rightarrow B \ell_{0} \mathbb{A}^{3}$ transforms to the conic bundle in $B \ell_{0} \mathbb{A}^{3} \times \mathbb{P}^{2}$ defined by

$$
x^{2}-u_{0} y^{2}-2 t_{0} y z-v_{0} z^{2}=0,
$$

obtained by base-change from the conic bundle with this defining equation over $\mathbb{A}^{3}$.

Proof. If $\mathcal{B}$ is the locally free coherent sheaf $\mathcal{O}_{B \ell_{0} \mathbb{A}^{3}}(1) \oplus \mathcal{O}_{B \ell_{0} \mathbb{A}^{3}} \oplus \mathcal{O}_{B \ell_{0} \mathbb{A}^{3}}$, the elementary transformation applied to $\mathbb{P}(\mathcal{B})$ with the indicated section over $E$ yields $\mathbb{P}\left(\mathcal{B}^{\prime}\right)$, where the dual $\mathcal{B}^{\prime \vee}$ sits in an exact sequence

$$
0 \rightarrow \mathcal{B}^{\prime \vee} \rightarrow \mathcal{B}^{\vee} \rightarrow \mathcal{O}_{E}(-1) \rightarrow 0 \text {. }
$$

So, $\mathcal{B}^{\prime} \cong \mathcal{O}_{B \ell_{0} \mathbb{A}^{3}}^{3}$. A direct computation establishes the remaining assertion.

Returning to the $G$-torsor over $X$ we are also interested in the vector bundles associated with the representations given in Section 5.1. Notation $R$ with subscript will be used for the associated vector bundle, where the subscript indicates the representation. When the generic stabilizer $\mathbb{Z} / 2 \mathbb{Z}$ of $X$ acts trivially on fibers, the vector bundle descends to $W$ and the notation $Q$ with subscript will be used for the vector bundle on $W$.

Lemma 15. Let $\Delta$ denote the diagonal in $\mathbb{P}^{1} \times \mathbb{P}^{1}$ and $r \in \Delta$, and let us use the same notation for the corresponding closed substacks of $W$, or of $X$.

(i) The line bundle $R_{\chi}$ is the pullback of line bundle $Q_{\chi}$ on $W$, with

$$
Q_{\chi} \cong \mathcal{O}_{W}\left(\Delta-H^{\prime}-H^{\prime \prime}\right)
$$

and characterization up to isomorphism as nontrivial, 2-torsion in $\operatorname{Pic}(W)$.

(ii) The vector bundle $R_{\rho_{2}}$ fits in an exact sequence

$$
0 \rightarrow M \rightarrow R_{\rho_{2}} \rightarrow M^{\vee} \otimes R_{\chi} \rightarrow M^{\vee} \otimes R_{\chi} \otimes \mathcal{O}_{\{r\}} \rightarrow 0
$$

of coherent sheaves on $X$.

Proof. There is no loss of generality in supposing $r$ to be the point of intersection of $H^{\prime}$ and $H^{\prime \prime}$.

The line bundle $R_{\chi}$ is the pullback of the nontrivial line bundle on $B \mathbb{Z} / 2 \mathbb{Z}$, corresponding to the $\mathbb{Z} / 2 \mathbb{Z}$-torsor

$$
\left(\mathbb{P}^{1} \times \mathbb{P}^{1}\right) \times_{\mathbb{P}^{2}} \sqrt{\mathcal{O}_{\mathbb{P}^{2}}(-H)} \rightarrow X .
$$


So $Q_{\chi}$ exists, corresponds analogously to the $\mathbb{Z} / 2 \mathbb{Z}$-torsor $\mathbb{P}^{1} \times \mathbb{P}^{1} \rightarrow W$, and is 2-torsion in $\operatorname{Pic}(W)$. As well, $Q_{\chi}$ is nontrivial, since we have at $r$ a copy of $B \mathbb{Z} / 2 \mathbb{Z}$ in $W$. By the general description of the Picard group of a root stack $[8, \S 3.1], \operatorname{Pic}(W)$ is generated by classes pulled back from $\mathbb{P}^{2}$ and the class of $\mathcal{O}_{W}(\Delta)$, a line bundle whose square is isomorphic to the pullback of $\mathcal{O}_{\mathbb{P}^{2}}(2)$. So there is a unique nontrivial 2-torsion class in $\operatorname{Pic}(W)$, the one indicated in the statement of (i).

For (ii), we start with the description of $R_{\rho_{2}}$ as isomorphic after pullback to $W$ to $L \oplus \sigma^{*} L$ and characterized by descent by the isomorphism $\sigma^{*}\left(L \oplus \sigma^{*} L\right) \cong L \oplus \sigma^{*} L$, as indicated in the description of $\rho_{2}$ in Section 5.1. In the present case, $L \oplus \sigma^{*} L$ contains a diagonal copy of $M^{\prime}$, yielding upon descent an injective homomorphism

$$
M \rightarrow R_{\rho_{2}}
$$

of coherent sheaves on $X$. The cokernel $K$ is locally free of rank 1 away from $r$. The reflexive hull $K^{\vee \vee}$ is a line bundle, whose isomorphism type is identified, by taking determinants, as $M^{\vee} \otimes R_{\chi}$. Furthermore, $K^{\vee \vee} /\left.K \cong K^{\vee \vee}\right|_{\{r\}}$. Combining this information, we obtain the 4-term exact sequence given in the statement.

Proposition 16. The following cohomology groups vanish:

$H^{i}\left(X, R_{\rho_{3}} \otimes \mathcal{O}_{X}\left(-H^{\prime}-H^{\prime \prime}\right)\right) \quad$ and $\quad H^{i}\left(X, R_{\rho_{3}} \otimes M\right) \quad$ for all $i$, and $H^{i}\left(X, R_{\rho_{3}} \otimes\left(M^{\vee}\right)^{\otimes j}\right)$ for all $i, j>0$.

Proof. The direct image functor on quasi-coherent sheaves is exact, for the morphisms $X \rightarrow W$ and $W \rightarrow \mathbb{P}^{2}$. So, each of the above cohomology groups may be identified with a cohomology group of the direct image sheaf on $\mathbb{P}^{2}$. Using Lemma 15, the direct image of $R_{\rho_{3}} \otimes$ $\mathcal{O}_{X}\left(-H^{\prime}-H^{\prime \prime}\right)$ on $W$ is $\mathcal{O}_{W}\left(\Delta-2 H^{\prime}-2 H^{\prime \prime}\right)$, and on $\mathbb{P}^{2}$ is $\mathcal{O}_{\mathbb{P}^{2}}(-2)$, which has vanishing cohomology groups. For $R_{\rho_{3}} \otimes M$ we have direct image on $W$ that sits in an exact sequence between $\mathcal{O}_{W}\left(-H^{\prime}-H^{\prime \prime}\right)$ and the kernel of restriction to the fiber over $r$ of $\mathcal{O}_{W}\left(\Delta-H^{\prime}-H^{\prime \prime}\right)$. But the stabilizer action on fiber over $r$ is nontrivial, hence the sheaf $\mathcal{O}_{W}\left(\Delta-H^{\prime}-H^{\prime \prime}\right)$ and its kernel of restriction to the fiber over $r$ have the same direct image on $\mathbb{P}^{2}$. Consequently, on $\mathbb{P}^{2}$ we obtain the sheaf whose cohomology we need to compute in the middle of a short exact sequence with $\mathcal{O}_{\mathbb{P}^{2}}(-1)$ on the left and on the right. The vanishing of cohomology groups follows. The computation is similar for $R_{\rho_{3}} \otimes\left(M^{\vee}\right)^{\otimes j}$ when $j$ is odd, except that $\mathcal{O}_{\mathbb{P}^{2}}(i)$ with some $i>0$ occurs left and right, and we obtain the vanishing of all higher cohomology 
groups. The case that $j$ is even reduces as well to the vanishing of higher cohomology of $\mathcal{O}_{\mathbb{P}^{2}}(i)$ for $i \geq 0$; we omit the details.

5.4. Deformation result. Working over an algebraically closed field $k$ of characteristic different from 2, we prove the existence of families of conic bundles, where the special fiber is a standard conic bundle over a rational surface with nodal discriminant curve, and in all other fibers the discriminant curve is smooth.

Theorem 17. Let $\left(B, b_{0}\right)$ be a pointed curve, $\mathcal{S} \rightarrow B$ a smooth projective family of rational surfaces, and $\mathcal{D} \subset \mathcal{S}$ an irreducible divisor, smooth except for ordinary double points at $q_{1}, \ldots, q_{r}$ in the fiber $\mathcal{S}_{b_{0}}$, for some positive integer $r$. Let $\widetilde{\mathcal{D}} \rightarrow \mathcal{D}$ be a finite morphism of degree 2 , étale over $\mathcal{D} \backslash\left\{q_{1}, \ldots, q_{r}\right\}$, with $\widetilde{\mathcal{D}}$ smooth. We suppose, further, that $\mathcal{D}_{b_{0}}$ is connected and smooth except for nodes at $q_{1}, \ldots, q_{r}$, and $\mathcal{D}_{b}$ is smooth for all $b \neq b_{0}$. Then, after replacing $\left(B, b_{0}\right)$ by an étale neighborhood, there exists a variety $\mathcal{V}$ fitting into a commutative diagram

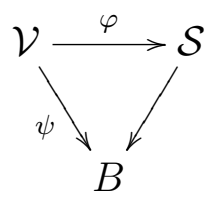

such that $\varphi$ is a conic bundle (flat, projective, generically smooth, all fibers are conics), $\psi$ is smooth, and for every $b \in B$ the fiber $\mathcal{V}_{b}$ is a standard conic bundle over $\mathcal{S}_{b}$ with corresponding ramification data $\widetilde{\mathcal{D}}_{b} \rightarrow \mathcal{D}_{b}$.

Remark 18. The proof starts by replacing the fiber $\mathcal{S}_{b_{0}}$ by a root stack of the sort defined by Matsuki and Olsson (cf. Section 5.2). In the envisaged application, $\mathcal{D}_{b_{0}}$ consists of two smooth curves meeting at the nodes $q_{1}, \ldots, q_{r}$. Then this root stack is just the iterated root stack of $\mathcal{S}_{b_{0}}$ along the two curves, and the subtle construction of Matsuki and Olsson, based on logarithmic structures, is not needed.

Proof. We follow the strategy of [13]. We construct, on the fiber over $c$, a suitable rank 2 vector bundle over a $\mu_{2}$-gerbe over a root stack of $\mathcal{S}_{b_{0}}$; this determines $\mathcal{V}_{b_{0}}$. We extend first the $\mu_{2}$-gerbe and then the vector bundle to the whole family, allowing ourselves at each step to replace $\left(B, b_{0}\right)$ by an étale neighborhood.

We denote the fiber $\mathcal{S}_{b_{0}}$ by $S$ and the fiber of the divisor and cover $\widetilde{\mathcal{D}}_{b_{0}} \rightarrow \mathcal{D}_{b_{0}}$ by $\widetilde{D} \rightarrow D$. Since $D$ is a curve with nodes at $q_{1}, \ldots, q_{r}$, the construction of Matsuki and Olsson mentioned in Section 5.2 yields a 
smooth Deligne-Mumford stack $Y$ with flat morphism to $S$ that is an isomorphism over $S \backslash D$. Over smooth points, respectively over nodes of $D$ the stack $Y$ has stabilizer $\mu_{2}$, respectively $\mu_{2} \times \mu_{2}$. The cover $\widetilde{D} \rightarrow D$ determines a 2-torsion element of $\operatorname{Br}(S \backslash D)$, which is the restriction of a unique element of $\operatorname{Br}(Y)$ (cf. [13, Prop. 2 and Prop. 5]). There is, correspondingly, a $\mu_{2}$-gerbe

$$
Z \rightarrow Y
$$

which we may take over smooth points, respectively over nodes of $D$ to have stabilizer $\mu_{2} \times \mu_{2}$, respectively the dihedral group $\mathfrak{D}_{4}$, with locally free sheaf $\mathcal{F}$ of rank 2 , such that the projectivization of $\mathcal{F}$ is base-change to $Z$ of a smooth $\mathbb{P}^{1}$-fibration over $Y$, birational to a conic bundle over $S$ with ramification data $\widetilde{D} \rightarrow D$.

By [13, Prop. 17], after replacing $\mathcal{F}$ by a suitable locally free subsheaf (obtained from $\mathcal{F}$ by elementary transformation over a smooth divisor in $Y$, which may be taken in general position with respect to the locus with nontrivial stabilizer), we may suppose that the kernel of the trace homomorphism

$$
H^{2}\left(Y,\left(\mathcal{F}^{\vee} \otimes \mathcal{F}\right)_{0}\right):=\operatorname{ker}\left(H^{2}\left(Y, \mathcal{F}^{\vee} \otimes \mathcal{F}\right) \rightarrow H^{2}\left(Y, \mathcal{O}_{Y}\right)\right)
$$

vanishes; although $\mathcal{F}$ is a sheaf on the gerbe $Z$, the locally free sheaf $\mathcal{F}^{\vee} \otimes \mathcal{F}$ descends to $Y$, and this is meant by the above notation. The space $H^{2}\left(Y,\left(\mathcal{F}^{\vee} \otimes \mathcal{F}\right)_{0}\right)$ is the obstruction space for the deformation theory of locally free coherent sheaves with given determinant.

The Deligne-Mumford stack $Y$ does not sit in a smooth family with root stacks $\sqrt{\left(\mathcal{S}_{b}, \mathcal{D}_{b}\right)}$. After suitable modification, however, it sits in a flat family.

The singularities of $\mathcal{D}$ are resolved by blowing up:

$$
\mathcal{S}^{\prime}:=B \ell_{\left\{q_{1}, \ldots, q_{r}\right\}} \mathcal{S} \quad \text { with smooth divisor } \quad \mathcal{D}^{\prime}:=B \ell_{\left\{q_{1}, \ldots, q_{r}\right\}} \mathcal{D} .
$$

Let $E=\bigcup_{i=1}^{r} E_{i}$ denote the exceptional divisor in $\mathcal{S}^{\prime}$. The divisors $\mathcal{D}^{\prime}$ and $E$ meet transversely, so the iterated root stack

$$
\mathcal{Y}^{\prime}:=\sqrt{\left(\mathcal{S}^{\prime},\left\{\mathcal{D}^{\prime}, E\right\}\right)}
$$

is smooth. As a family over $B$, this has nonreduced fiber $\mathcal{Y}_{b_{0}}^{\prime}$, but

$$
\left(\mathcal{Y}_{b_{0}}^{\prime}\right)_{\mathrm{red}}=Y^{\prime} \cup\left(\bigcup_{i=1}^{r} X_{i}\right),
$$

where $Y^{\prime}$ denotes the blow-up of $Y$ at the points over $q_{1}, \ldots, q_{r}$, and each $X_{i}$ is a copy of the stack $X$ glued along the locus defined by the vanishing of the coordinate $t$, in the notation of Section 5.3. Let $Z^{\prime}$ 
denote the corresponding blow-up of $Z$, so $Z^{\prime} \cong Y^{\prime} \times_{Y} Z$. The coarse moduli space of $\left(\mathcal{Y}_{b_{0}}^{\prime}\right)_{\text {red }}$ is $\mathcal{S}_{b_{0}}^{\prime}=S^{\prime} \cup\left(\bigcup_{i=1}^{r} E_{i}\right)$, where $S^{\prime}$ denotes the blow-up of $S$ at $q_{1}, \ldots, q_{r}$.

Over $X$ there is the $G$-torsor introduced in Section 5.3, where $G$ is the algebraic group, defined and exhibited as a $\mu_{2}$-extension of itself in Section 5.1. This way, we get a $\mu_{2}$-gerbe $U \rightarrow X$. The substack in $X$ defined by $t=0$ is isomorphic to $\left[\mathbb{P}^{1} /(\mathbb{Z} / 2 \mathbb{Z})\right] \times B \mathbb{Z} / 2 \mathbb{Z}$, and the restriction of the $G$-torsor admits the description of Example 11. Consequently, the restriction of $U$ is isomorphic to $\left[\mathbb{P}^{1} / K\right]$, where $K$ denotes the $\mu_{2}$-extension of the copy of the Klein four-group in $G$ generated by the $2 \times 2$-permutation matrices and $\mu_{2}$, acting through the Klein fourgroup. Each exceptional divisor of $Z^{\prime}$ admits the same description. So, we may glue to obtain

$$
Z^{\prime} \cup\left(\bigcup_{i=1}^{r} U_{i}\right)
$$

a $\mu_{2}$-gerbe over $\left(\mathcal{Y}_{b_{0}}^{\prime}\right)_{\text {red }}$, where each $U_{i}$ is a copy of $U$. By applying the proper base change theorem for tame Deligne-Mumford stacks [1, App. A] to the corresponding class

$$
\gamma \in H^{2}\left(\mathcal{Y}_{b_{0}}^{\prime}, \mu_{2}\right)=H^{2}\left(\left(\mathcal{Y}_{b_{0}}^{\prime}\right)_{\mathrm{red}}, \mu_{2}\right)
$$

we obtain as in [13] a class

$$
\Gamma \in H^{2}\left(\mathcal{Y}^{\prime}, \mu_{2}\right)
$$

extending $\gamma$. Accordingly there is a $\mu_{2}$-gerbe

$$
\mathcal{Z}^{\prime} \rightarrow \mathcal{Y}^{\prime}
$$

with

$$
\left(\mathcal{Z}_{b_{0}}^{\prime}\right)_{\text {red }} \cong Z^{\prime} \cup\left(\bigcup_{i=1}^{r} U_{i}\right) .
$$

Corresponding to the representation $\rho_{2}$ of $G$ is a rank 2 vector bundle on $U$. The restriction to the copy of $\left[\mathbb{P}^{1} / K\right]$ over $t=0$ is determined by the 2-dimensional representation of $K \subset G$. On the other hand, the restriction of $\mathcal{F}$ over a point $q_{i}$ is associated with a linear representation of $\mathfrak{D}_{4}$, which after blowing up becomes $\left[\mathbb{P}^{1} / \mathfrak{D}_{4}\right]$ over the exceptional divisor, in a manner that is compatible under $\mathfrak{D}_{4} \cong K$. By identifying restrictions in this manner, we obtain a rank 2 vector bundle $\mathcal{F}_{0}^{\prime}$ on $\left(\mathcal{Z}_{b_{0}}^{\prime}\right)_{\text {red }}$ from the pullback $\mathcal{F}^{\prime}$ of $\mathcal{F}$ under $Z^{\prime} \rightarrow Z$ and the rank 2 vector bundle on $U_{i}$ for every $i$. Its determinant descends to a line bundle on $\left(\mathcal{Y}_{b_{0}}^{\prime}\right)_{\text {red. }}$ 
We argue that the determinant line bundle on $\left(\mathcal{Y}_{b_{0}}^{\prime}\right)_{\text {red }}$ extends to a line bundle on $\mathcal{Y}^{\prime}$, after replacing $\left(B, b_{0}\right)$ by a suitable étale neighborhood. It suffices to verify this after tensoring by the restriction of a line bundle on $\mathcal{Y}^{\prime}$. Over $\mathcal{D}^{\prime}$ there is a divisor in $\mathcal{Y}^{\prime}$; we tensor by the restriction of the associated line bundle. This yields a line bundle on $\left(\mathcal{Y}_{b_{0}}^{\prime}\right)_{\text {red }}$ with trivial stabilizer actions. By [4, Thm. 10.3], this is isomorphic to the pullback of a line bundle on the coarse moduli space $\mathcal{S}_{b_{0}}^{\prime}$, It thus suffices to show that every line bundle on $\mathcal{S}_{b_{0}}^{\prime}$ extends (after replacing $\left(B, B_{0}\right)$ by a suitable étale neighborhood) to a line bundle on $\mathcal{S}^{\prime}$. Tensoring by line bundles associated with the $E_{i}$ with appropriate multiplicities, we are reduced to considering line bundles on $\mathcal{S}_{b_{0}}^{\prime}$ pulled back from $S$, and thus to the corresponding fact for line bundles on $\mathcal{S}$ and $S$, which is standard since $\mathcal{S}$ is a smooth projective family of rational surfaces.

Now we show that the obstructions to extending $\mathcal{F}_{0}^{\prime}$ to a vector bundle on $\mathcal{Z}^{\prime}$ vanish. We define

$$
\left(\mathcal{Z}_{p}^{\prime}\right)_{\text {red }}=T_{0} \subset T_{0}^{\prime} \subset T_{0}^{\prime \prime} \subset T_{1} \subset T_{1}^{\prime} \subset \ldots
$$

to be the substacks of $\mathcal{Z}^{\prime}$ corresponding to the following effective divisors:

\begin{tabular}{l|l|l} 
& divisor & obstruction space \\
\hline$T_{i}$ & $(2 i+1)\left(X_{1}+\cdots+X_{r}\right)+(i+1) Y^{\prime}$ & $H^{2}\left(X, R_{\rho_{3}} \otimes M\right)^{r}$ \\
$T_{i}^{\prime}$ & $(2 i+2)\left(X_{1}+\cdots+X_{r}\right)+(i+1) Y^{\prime}$ & $H^{2}\left(Y^{\prime},\left(\mathcal{F}^{\prime \vee} \otimes \mathcal{F}^{\prime}\right)_{0}\right)$ \\
$T_{i}^{\prime \prime}$ & $(2 i+2)\left(X_{1}+\cdots+X_{r}\right)+(i+2) Y^{\prime}$ & $H^{2}\left(X, R_{\rho_{3}} \otimes \mathcal{O}_{X}\left(-H^{\prime}-H^{\prime \prime}\right)\right)^{r}$
\end{tabular}

In each case we have identified the obstruction space to extension of locally free sheaf (on the gerbe) with fixed determinant. The vanishing in each cases follows from Proposition 16 (cohomology on $X$ ) or the vanishing of $H^{2}\left(Y,\left(\mathcal{F}^{\vee} \otimes \mathcal{F}\right)_{0}\right)$. So, starting with $\mathcal{F}_{0}^{\prime}$ on the gerbe over $T_{0}$, we may extend to successive infinitesimal neighborhoods and, by appealing as in [13] to the Grothendieck existence theorem for tame Deligne-Mumford stacks [3, Thm. A.1.1], to a locally free sheaf $\widetilde{\mathcal{F}}^{\prime}$ on $\mathcal{Z}^{\prime}$ extending $\mathcal{F}_{0}^{\prime}$.

Associated with $\widetilde{\mathcal{F}}^{\prime}$ is a smooth $\mathbb{P}^{1}$-fibration

$$
\mathcal{P}^{\prime} \rightarrow \mathcal{Y}^{\prime}
$$

The conic bundle construction of Section 5.2 yields a standard conic bundle

$$
\mathcal{V}^{\prime} \rightarrow \mathcal{S}^{\prime}
$$

We claim that with an elementary transformation and contraction, from $\mathcal{V}^{\prime} \rightarrow \mathcal{S}^{\prime}$ we obtain $\mathcal{V} \rightarrow \mathcal{S}$, fulfilling the requirements of the 
theorem. We have already seen this in Proposition 14 for the conic bundle $V \rightarrow B \ell_{0} \mathbb{A}^{3}$. So, to complete the proof, it is enough to show for every $i$ that $q_{i} \in \mathcal{S}$ and $0 \in \mathbb{A}^{3}$ have a common étale neighborhood, over which $\mathcal{V}^{\prime} \rightarrow \mathcal{S}^{\prime}$ and $V \rightarrow B \ell_{0} \mathbb{A}^{3}$ are isomorphic.

Since $\mathcal{D}$ has an ordinary double point at $q_{i}$, there is a diagram of étale morphisms of pointed schemes

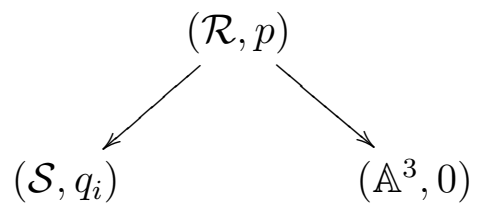

such that $\mathcal{D} \subset \mathcal{S}$ and the divisor in $\mathbb{A}^{3}$ defined by $u_{0} v_{0}-t_{0}^{2}=0$ have the same pre-image in $\mathcal{R}$. Without loss of generality, $p$ is the only point of $\mathcal{R}$ mapping to $q_{i}$ in $\mathcal{S}$, and is the only point of $\mathcal{R}$ mapping to 0 in $\mathbb{A}^{3}$. As well, we may suppose that the image of the left-hand morphism avoids $q_{j}$ for all $j \neq i$. After blowing up, we obtain an analogous diagram with étale morphisms

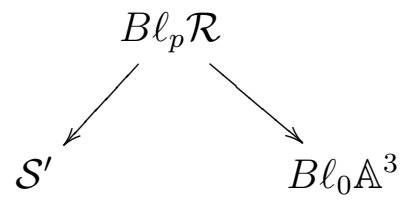

There is a further diagram, in which each scheme is replaced by the iterated root stack along the proper transform of the given divisor and the exceptional divisor.

The class $\Gamma \in H^{2}\left(\mathcal{Y}^{\prime}, \mu_{2}\right)$ pulls back to a class in $H^{2}$ of the iterated root stack of $B \ell_{p} \mathcal{R}$. As well, the $G$-torsor over $X$, pulled back to $M$ (Section 5.3), pulls back to a class in the same $H^{2}$ group. Since $\Gamma$ extends $\gamma$, whose construction also is based on the $G$-torsor over $X$ of Section 5.3, we may conclude by applying the proper base change theorem to the morphism to $\mathcal{R}$, that after possibly replacing $(\mathcal{R}, p)$ by an étale neighborhood, these two $H^{2}$-classes coincide. So, we obtain another analogous diagram of $\mu_{2}$-gerbes over iterated root stacks.

Now we compare the vector bundles, obtained by pullback from $\widetilde{\mathcal{F}}^{\prime}$ on $\mathcal{Z}^{\prime}$ and the rank 2 vector bundle on the $\mu_{2}$-gerbe over $M$, again coming from the $G$-torsor over $M$ in Section 5.3. Their restrictions to the copy of $U$ over $p$ are, again by construction, isomorphic. By an argument, analogous to above obstruction analysis but this time using the vanishing of $H^{1}$, we see that an isomorphism over the copy of $U$ may be extended to all infinitesimal neighborhoods. So, by the 
standard combination of the Grothendieck existence theorem and Artin approximation, after replacing $(\mathcal{R}, p)$ by an étale neighborhood there is an isomorphism of the two vector bundles over the gerbe over the iterated root stack of $B \ell_{p} \mathcal{R}$. Consequently, the associated smooth $\mathbb{P}^{1}$ fibrations are isomorphic. But the conic bundle construction of Section 5.2 commutes with étale base change of the underlying algebraic variety. So the conic bundles $\mathcal{V}^{\prime} \times_{\mathcal{S}^{\prime}} B \ell_{p} \mathcal{R}$ and $V \times_{B \ell_{0} \mathbb{A}^{3}} B \ell_{p} \mathcal{R}$ are isomorphic over $B \ell_{p} \mathcal{R}$, as required.

\section{Proof of Theorem 1}

Let $\mathcal{S} \rightarrow B$ be as in Section 4.2. If $\mathcal{D} \rightarrow B$ is chosen in a general manner, then $\mathcal{D}$ will have smooth total space. Note that after replacing $B$ by simply branched cover, $\mathcal{D}$ will satisfy the hypotheses in Theorem 17 . So, we may apply Theorem 17 to obtain a family $\mathcal{V} \rightarrow \mathcal{S} \rightarrow B$. The composite $\mathcal{V} \rightarrow B$ is smooth, and $\mathcal{V}_{b_{0}}$ is stably rational; it remains to show that the very general fiber is not stably rational.

We recall the main result of [13]:

Theorem 19. Let $\mathcal{L}$ be a linear system of effective divisors on a smooth projective rational surface $S$, with smooth and irreducible general member. Let $\mathcal{M}$ be an irreducible component of the moduli space of pairs $(D, \tilde{D} \rightarrow D)$, where $D \in \mathcal{L}$ is nodal and reduced and $\tilde{D} \rightarrow D$ is an étale cover of degree 2. Assume that $\mathcal{M}$ contains a cover that is nontrivial over every irreducible component of a reducible curve with smooth irreducible components. Then a conic bundle over $S$ corresponding to a very general point of $\mathcal{M}$ is not stably rational.

We seek to apply this to standard conic bundles $V \rightarrow S$ over a quintic del Pezzo surface with degeneracy locus a generic bi-anticanonical divisor, as in Section 4.3. The relevant reducible curve is a union of two generic anticanonical divisors, i.e., elliptic curves which admit nontrivial étale double covers. The birational class of $V$ depends on the choice of a non-trivial étale double cover $\tilde{D} \rightarrow D$. We can invoke Theorem 19 once we know that the monodromy action on such double covers is transitive.

We apply the criterion of [6, Thm. 3]; see also [12, Sect. 3]. We need to find members $D, D^{\prime} \in\left|-2 K_{S}\right|$ such that

- $D$ has an $E_{6}$ singularity;

- $D^{\prime}$ is a union of a pair of smooth curves meeting transversally in an odd number of points. 
The second is obvious - again use a pair of anticanonical curves. An $E_{6}$ singularity is locally analytically equivalent to $y^{3}=x^{4}$. The plane sextic

$$
\begin{array}{r}
\frac{1}{2} y^{4} z^{2}+y^{3} z^{3}+\frac{1}{2} x y^{4} z+x y^{3} z^{2}-x^{2} y^{3} z-3 x^{2} y^{2} z^{2}-x^{3} y^{3}-2 x^{3} y^{2} z+ \\
\frac{1}{2} x^{3} y z^{2}+3 x^{4} y^{2}-\frac{1}{2} x^{4} y z+x^{4} z^{2}=0
\end{array}
$$

has $E_{6}$-singularity at $(0: 0: 1)$ and nodes at four other general points, hence defines such a curve $D$.

\section{Limitations OF THIS CONSTRUCTION}

The stable rationality of smooth cubic threefolds is quite mysterious. No stably rational examples are known but the known arguments for disproving stable rationality fail in this case. Voisin [33] has shown that the existence of a decomposition of the diagonal for a cubic threefold $X$ reduces to finding curves of 'odd degree' in its intermediate Jacobian $\mathrm{IJ}(X)$. Such curves arise in many examples, which are therefore natural candidates for stable rationality.

We would have liked to use the approach of Section 3 to exhibit a stably rational cubic threefold. Following the procedure of Section 4.1, for a nonsingular plane cubic $C \subset \mathbb{P}^{2}$ and point $p:=s_{4} \in \mathbb{P}^{2} \backslash C$, we would need to have $p \in L$. Yet $L$ must be the satellite conic, which contradicts Lemma 8.

\section{REFERENCES}

[1] D. Abramovich, A. Corti, and A. Vistoli. Twisted bundles and admissible covers. Comm. Algebra, 31(8):3547-3618, 2003. Special issue in honor of Steven L. Kleiman.

[2] D. Abramovich, T. Graber, and A. Vistoli. Gromov-Witten theory of DeligneMumford stacks. Amer. J. Math., 130(5):1337-1398, 2008.

[3] D. Abramovich and A. Vistoli. Compactifying the space of stable maps. $J$. Amer. Math. Soc., 15(1):27-75, 2002.

[4] J. Alper. Good moduli spaces for Artin stacks. Ann. Inst. Fourier (Grenoble), 63(6):2349-2402, 2013.

[5] M. Artin and D. Mumford. Some elementary examples of unirational varieties which are not rational. Proc. London Math. Soc. (3), 25:75-95, 1972.

[6] A. Beauville. Le groupe de monodromie des familles universelles d'hypersurfaces et d'intersections complètes. In Complex analysis and algebraic geometry (Göttingen, 1985), volume 1194 of Lecture Notes in Math., pages 8-18. Springer, Berlin, 1986. 
[7] A. Beauville, J.-L. Colliot-Thélène, J.-J. Sansuc, and P. Swinnerton-Dyer. Variétés stablement rationnelles non rationnelles. Ann. of Math. (2), 121(2):283318, 1985.

[8] C. Cadman. Using stacks to impose tangency conditions on curves. Amer. J. Math., 129(2):405-427, 2007.

[9] J.-L. Colliot-Thélène and A. Pirutka. Hypersurfaces quartiques de dimension 3: non-rationalité stable. Ann. Sci. Éc. Norm. Supér. (4), 49(2):371-397, 2016.

[10] I. V. Dolgachev. Classical algebraic geometry: A modern view. Cambridge University Press, Cambridge, 2012.

[11] J. Hall and D. Rydh. Coherent Tannaka duality and algebraicity of Homstacks, 2016. arXiv:1405.7680.

[12] B. Hassett, A. Kresch, and Yu. Tschinkel. On the moduli of degree 4 Del Pezzo surfaces. In Development of moduli theory (Kyoto, 2013), volume 69 of Adv. Stud. Pure Math., pages 349-386. Math. Soc. Japan, Tokyo, 2016.

[13] B. Hassett, A. Kresch, and Yu. Tschinkel. Stable rationality and conic bundles. Math. Ann., 365(3-4):1201-1217, 2016.

[14] B. Hassett, A. Pirutka, and Yu. Tschinkel. Stable rationality of quadric surface bundles over surfaces. Acta Math., to appear, 2016. arXiv:1603.09262.

[15] B. Hassett, A. Pirutka, and Yu. Tschinkel. Intersections of three quadrics in $\mathbb{P}^{7}, 2017$. arXiv: 1706.01371.

[16] B. Hassett and Yu. Tschinkel. On stable rationality of Fano threefolds and del Pezzo fibrations. J. Reine Angew. Math., to appear, 2016. arXiv:1601.07074.

[17] M. Kontsevich and Yu. Tschinkel. Specialization of birational types, 2017. arXiv: 1708.05699.

[18] A. Kresch and Yu. Tschinkel. Models of Brauer-Severi surface bundles, 2017. arXiv: 1708.06277.

[19] B. Ė. Kunyavskiı̌, A. N. Skorobogatov, and M. A. Tsfasman. Del Pezzo surfaces of degree four. Mém. Soc. Math. France (N.S.), (37):113, 1989.

[20] M. Maruyama. On a family of algebraic vector bundles. In Number theory, algebraic geometry and commutative algebra, in honor of Yasuo Akizuki, pages 95-146. Kinokuniya, Tokyo, 1973.

[21] K. Matsuki and M. Olsson. Kawamata-Viehweg vanishing as Kodaira vanishing for stacks. Math. Res. Lett., 12(2-3):207-217, 2005.

[22] C. T. McMullen, R. E. Mukamel, and A. Wright. Cubic curves and totally geodesic subvarieties of moduli space. Ann. of Math. (2), 185(3):957-990, 2017.

[23] J. Nicaise and E. Shinder. The motivic nearby fiber and degeneration of stable rationality, 2017. arXiv: 1708.02790.

[24] J. Oesinghaus. Conic bundles and iterated root stacks, 2017. arXiv: 1709.08552.

[25] V. G. Sarkisov. Birational automorphisms of conic bundles. Izv. Akad. Nauk SSSR Ser. Mat., 44(4):918-945, 974, 1980.

[26] V. G. Sarkisov. On conic bundle structures. Izv. Akad. Nauk SSSR Ser. Mat., 46(2):371-408, 432, 1982.

[27] S. Schreieder. On the rationality problem for quadric bundles, 2017. arXiv: 1706.01356. 
[28] S. Schreieder. Quadric surface bundles over surfaces and stable rationality, 2017. arXiv: 1706.01358.

[29] N. I. Shepherd-Barron. Stably rational irrational varieties. In The Fano Conference, pages 693-700. Univ. Torino, Turin, 2004.

[30] B. Totaro. Hypersurfaces that are not stably rational. J. Amer. Math. Soc., 29(3):883-891, 2016.

[31] Yu. Tschinkel and K. Yang. Potentially stably rational del Pezzo surfaces over nonclosed fields, 2018. cims.nyu.edu/ tschinke/papers/yuri/18h1dp/magma.

[32] C. Voisin. Unirational threefolds with no universal codimension 2 cycle. Invent. Math., 201(1):207-237, 2015.

[33] C. Voisin. On the universal $\mathrm{CH}_{0}$ group of cubic hypersurfaces. J. Eur. Math. Soc. (JEMS), 19(6):1619-1653, 2017.

Department of Mathematics, Brown University, 151 Thayer Street, Providence, Rhode IsLand 02912, USA

E-mail address: bhassett@math.brown.edu

Institut FÜr MATHEMATIK, UniversitÄt ZÜRICH, WinterthuRERSTRASSE 190, CH-8057 ZÜRICH, SwitzerLAND

E-mail address: andrew.kresch@math.uzh.ch

Courant Institute, New York University, New York, NY 10012, USA

E-mail address: tschinkel@cims.nyu.edu

Simons Foundation, 160 Fifth Avenue, New York, NY 10010, USA 